\section{Electrochemistry and electrogenerated chemiluminescence of thiophene and fluorene oligomers. Benzoyl peroxide as a coreactant for oligomerization of thiophene dimers $\uparrow$}

\author{
Alexander B. Nepomnyashchii, Robert J. Ono, Dani M. Lyons, Christopher W. Bielawski, Jonathan L. Sessler \\ and Allen J. Bard*
}

Received 2nd March 2012, Accepted 1st May 2012

DOI: $10.1039 / \mathrm{c} 2 \mathrm{sc20263h}$

\begin{abstract}
The electrochemical properties of oligomers of thiophene (with number of monomer units, $n$, from 2 to 12 ) and fluorene ( $n=3$ to 7 ) were investigated. Both sets of oligomers were characterized by the presence of two oxidation and two reduction waves as determined by cyclic voltammetry (CV), with the reversibility of the waves depending on the structural properties of the compounds. The addition or removal of a third electron was found to be difficult relative to the second, a finding shown for conjugated oligomers with chain lengths up to 7 in the case of the fluorenes and up to 12 for the thiophenes. The oligothiophenes showed a larger separation between the electrochemical waves for the same chain length, and also substantial electrogenerated chemiluminescence (ECL) signals, whose intensity increased with oligomer size. In contrast, the ECL intensity of the fluorene oligomers was essentially independent of chain length. The ECL spectra for the thiophene dodecamer were obtained with concentrations as low as $20 \mathrm{pM}$, a result that reflects a high ECL efficiency, close to that of the well-known ECL standard Ru(bpy) ${ }_{3}{ }^{2+}$. Oligomers were also formed on electrochemical reduction of an appropriately functionalized dimer in the presence of benzoyl peroxide producing a longer wavelength emission (maximum at $\sim 540 \mathrm{~nm}$ ) as opposed to the spectrum of the dimer $\left(\lambda_{\mathrm{em}}=\right.$ $390 \mathrm{~nm})$.
\end{abstract}

\section{Introduction}

We describe here the results of cyclic voltammetry (CV) analyses of various thiophene and fluorene oligomers and show that the potentials for stepwise oxidation and reduction correlate with the size of the molecules and the extent of conjugation. We also detail the electrogenerated chemiluminescence (ECL) features of these molecules. Electrochemical characterization of oligomers and polymers started about 35 years ago, ${ }^{1,2}$ with a special interest in the potentials needed for successive electron transfers (ET) from or into electroactive groups in the polymers. ${ }^{3-8}$ These ET reactions affect the conductivity and spectroscopic properties of the material and also provide information about the nature of the interactions among the electroactive groups on the polymer. ${ }^{9-18}$ The earliest electrochemical measurements involving macromolecules were carried out on polyvinyl polymers (e.g., redoxactive poly(vinylferrocene)s), which showed a response that was characteristic of no electronic interactions between neighboring groups (i.e., all the electrons were transferred in a single wave). ${ }^{3}$

University of Texas at Austin, 1 University Station A5300, Austin, TX, USA.E-mail: ajbard@mail.utexas.edu; Fax: +512 471 0088; Tel: +512 4713761

$\dagger$ Electronic supplementary information (ESI) available: Additional scan figures. See DOI: $10.1039 / \mathrm{c} 2 \mathrm{sc} 20263 \mathrm{~h}$
However polymers with interactions among the electroactive groups, e.g., where addition of an electron makes addition of an electron to a neighboring group more difficult, can show a series of waves for each sequential ET process. ${ }^{9-11}$ For example, in conjugated polymers, there are very strong interactions between neighbouring units, at least over a certain segment size. Much less is understood about sequential ET in oligomers as well as the rules that govern the splitting between sequential waves. ${ }^{19}$ In addition to characterization, many electrochemical investigations relate to the electrochemical preparation of polymers and the factors that affect their behavior. ${ }^{19}$

In this study we use well-defined oligomers of thiophene and fluorene as surrogates for conjugated polymers, which are widely investigated for their potential use in many practical applications. ${ }^{20-26}$ Much of the current attention centered on thiopheneand fluorene-derived polymers stems from their unique optical properties and their possible use in organic light-emitting and photovoltaic devices. ${ }^{26,27}$ Although the first conjugated polymers were synthesized more than 30 years ago, their structure, composition and behavior are still being actively investigated. Spectroscopically, the large size of these molecules and their stable fluorescence make them suitable for single molecule studies. ${ }^{28-30}$ For example, the presence of ordered, cylindrical conformations have been proposed for the poly(phenylenevinylene) system, 
a finding that has been rationalized in terms of short range emission localized within so-called "quasi-straight-chain" fragments of 5 to 20 units. $^{31}$

Electrochemical studies of large polymers are, however, hampered by small diffusion coefficients and low concentrations in convenient solvents, as well as polydispersity. Operationally, this results in small currents for stepwise processes. Thus, for fundamental studies, it is better to use pure, monodisperse molecules (i.e., discrete oligomers). If appropriately designed, such species could be useful in uncovering correlations between polymer structure and oxidation/reduction potential. To date, there have been a number of electrochemical studies of monodisperse oligomers, as well as polymers with up to 96 repeating units. $^{32-37}$ Studies of electrochemical behavior of oligomers of phenylvinylenes, boron dipyrromethene (BODIPY) dyes, thiophenes, fluorenes, and spirofluorenes show the presence of more than one wave for both oxidation and reduction. ${ }^{10,11,15,16,19,38,39}$ However, the separations between waves for successive electron transfers have not been delineated very clearly; neither have the differences between oxidation and reduction processes. In this work we attempt to establish guidelines for the separation between electrochemical waves for a range of thiophene and fluorene oligomer lengths. The thiophenes and fluorenes used in these studies are shown in Scheme 1. Substitution of the $\alpha$ - and $\alpha^{\prime}$-positions in the thiophenes was used to prevent chemical oxidation through these positions. ${ }^{40,41}$

Electrogenerated chemiluminescence (ECL) properties are also investigated to evaluate the effect of the structure and chain length on the wavelength and intensity of the emitted light. Previous studies for BODIPY molecules showed a substantial decrease of the ECL signal with size from monomer to dimer, trimer and polymer. ${ }^{11}$ The decrease of the signal was correlated with a decrease in the fluorescence intensity. This work demonstrates different effects of chain length on the ECL intensity for thiophene and fluorene oligomers. We also show that thiophenes dimerize on reduction in ECL studies. The use of ECL emission has been shown to be a useful technique in studies of dimerization and the formation of coupling products. ${ }^{42-45}$

\section{Experimental}

1-Bromo-2-hexyldecane, ${ }^{46} \quad 5,5^{\prime}$-dibromo-2, $2^{\prime}$-bithiophene, ${ }^{47}$ tdimer-2, ${ }^{48}$ t-trimer-1, ${ }^{48}$ t-trimer-2, ${ }^{49}$ t-tetramer, ${ }^{48}$ t-octamer, ${ }^{50} \mathrm{t}$ dodecamer ${ }^{50}$ were synthesized according to literature procedures. Thiophene t-dimer-1, t-hexamer-1, electrochemical-grade anhydrous MeCN (99.93\%), dichloromethane (DCM) (99.8\%), 99\% benzoyl peroxide, and tetra- $n$-butylammonium hexafluorophosphate $\left(\mathrm{TBAPF}_{6}\right)$ were purchased from Sigma-Aldrich Chemical Co. (Milwaukee, WI). Fluorene trimer, pentamer and heptamer were purchased from American Dye Source Inc. (Baie D'urfe, Quebec, Canada). All other chemicals were purchased from Sigma-Aldrich or Alfa Aesar (Ward Hill, MA) and used as received. Solvents for synthesis were dried using a Vacuum Atmospheres (Hawthorne, CA) solvent purification system or were distilled using standard methods. ${ }^{51}$ All reactions were carried out under an atmosphere of nitrogen. High resolution mass spectra (HRMS) were obtained by chemical ionization (CI) using a VG analytical ZAB2-E spectrometer. NMR spectra were

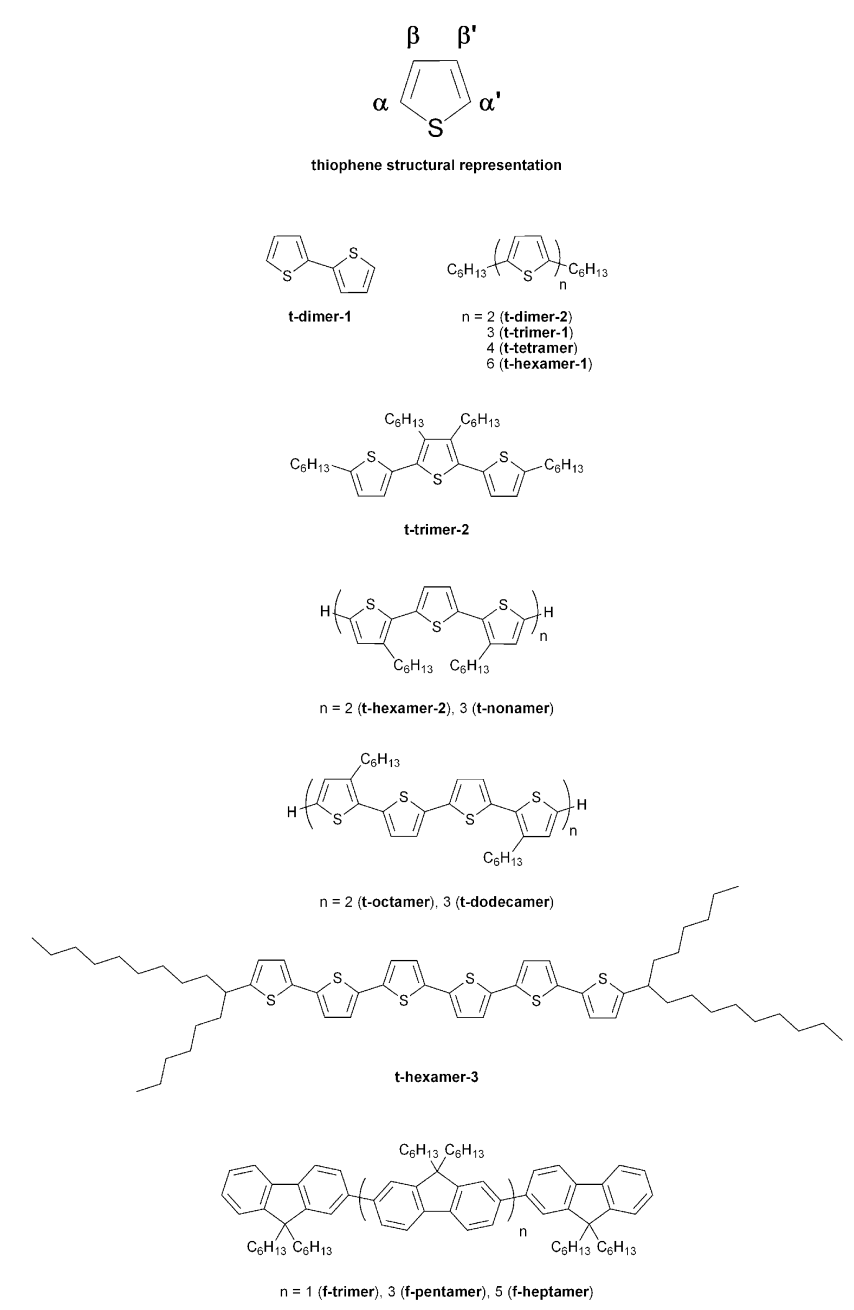

Scheme 1 Chemical structures of various thiophene and fluorene oligomers examined.

obtained on a Varian Unity INOVA $400 \mathrm{MHz}$ spectrometer in $\mathrm{CDCl}_{3}$ containing TMS as an internal reference.

\subsection{Synthesis}

5-(2-Hexyldecyl)-2,2'-bithiophene. To an oven dried Schlenk flask was added 2,2'-bithiophene $(2.00 \mathrm{~g}, 12.0 \mathrm{mmol})$ and THF $(30 \mathrm{~mL})$. The solution was cooled to $-70{ }^{\circ} \mathrm{C}$, and $n$-butyllithium in hexanes $(4.30 \mathrm{~mL}, 2.8 \mathrm{M})$ was added dropwise via syringe. After $1 \mathrm{~h}$ of stirring the solution was warmed to $-20{ }^{\circ} \mathrm{C}$, and 1 bromo-2-hexyldecane (3.66 g, $12.0 \mathrm{mmol}$ ) was added in one portion. The solution was warmed to ambient temperature and stirred for $4 \mathrm{~h}$, at which point a condenser was fitted and the mixture was heated to reflux overnight. Upon cooling the reaction mixture was quenched with $5 \mathrm{~mL}$ of $6 \mathrm{~N} \mathrm{HCl}$, and was extracted into hexanes. The solution was washed with deionized water and brine, dried $\left(\mathrm{Na}_{2} \mathrm{SO}_{4}\right)$ and the solvent was removed in vacuo. Subjecting the crude mixture to column chromatography (silica gel; hexanes) afforded the desired product as a yellow-green oil (1.74 g; 37\% yield). ${ }^{1} \mathrm{H}$ NMR $\left(\mathrm{CDCl}_{3}\right.$, $400 \mathrm{MHz}): \delta 0.87(\mathrm{t}, J=6.0 \mathrm{~Hz}, 6 \mathrm{H}), 1.25-1.29$ (br, 24H), 1.61 (br m, 1H), $2.71(\mathrm{~d}, J=6.4 \mathrm{~Hz}, 2 \mathrm{H}), 6.63(\mathrm{~d}, J=3.6 \mathrm{~Hz}, 1 \mathrm{H}), 6.98$ 
$(\mathrm{m}, 2 \mathrm{H}), 7.08(\mathrm{~d}, J=2.8 \mathrm{~Hz}, 1 \mathrm{H}), 7.14(\mathrm{~d}, J=4.0 \mathrm{~Hz}, 1 \mathrm{H}) .{ }^{13} \mathrm{C}$ NMR $\left(\mathrm{CDCl}_{3}, 100 \mathrm{MHz}\right): \delta$ 14.1, 22.7, 26.6, 26.7, 29.4, 29.6, 29.9, 31.91, 33.2, 34.5, 37.9, 38.0, 39.9, 122.9, 123.3, 123.6, 125.8, 127.6, 134.9, 138.0, 143.9. HRMS $m / z$ calcd for $\mathrm{C}_{24} \mathrm{H}_{38} \mathrm{~S}_{2}[\mathrm{M}+$ $\mathrm{H}]^{+}:$390.2415; found: 390.2415 .

5'-Bromo-5-(2-hexyldecyl)-2,2'-bithiophene. 5-(2-Hexyldecyl)$2,2^{\prime}$-bithiophene $(0.737 \mathrm{~g}, 1.89 \mathrm{mmol})$ was dissolved in dichloromethane $(10 \mathrm{~mL})$ and was stirred at ambient temperature. $N$-bromosuccinimide $(0.336 \mathrm{~g}, 1.89 \mathrm{mmol})$ was added portion-wise, and the resulting solution was stirred overnight at ambient temperature in the dark. The solution was filtered, and the resulting filtrate washed successively with $10 \% a q . \mathrm{KOH}$, deionized water, and brine, and was dried $\left(\mathrm{Na}_{2} \mathrm{SO}_{4}\right)$. The solvent was removed in vacuo and the crude mixture was subjected to column chromatography (silica gel; hexanes) to afford the desired product as a yellow oil (0.823 g; 93\% yield). ${ }^{1} \mathrm{H}$ NMR $\left(\mathrm{CDCl}_{3}, 400 \mathrm{MHz}\right): \delta 0.88(\mathrm{t}, J=6.8 \mathrm{~Hz}, 6 \mathrm{H}), 1.25-1.28(\mathrm{br}$, $24 \mathrm{H}), 1.62(\mathrm{~m}, 1 \mathrm{H}), 2.70(\mathrm{~d}, J=6.8 \mathrm{~Hz}, 2 \mathrm{H}), 6.62(\mathrm{~d}, J=3.6 \mathrm{~Hz}$, $1 \mathrm{H}), 6.82(\mathrm{~d}, J=4.0 \mathrm{~Hz}, 1 \mathrm{H}), 6.91(\mathrm{~m}, 2 \mathrm{H}) .{ }^{13} \mathrm{C} \mathrm{NMR}\left(\mathrm{CDCl}_{3}\right.$, $100 \mathrm{MHz}): \delta 14.1,22.7,26.6,29.3,29.62,29.9,31.9,33.2,34.5$, 39.9, 110.1, 122.9, 123.6, 125.8, 130.4, 133.9, 139.5, 144.5. HRMS $m / z$ calcd for $\mathrm{C}_{24} \mathrm{H}_{37} \mathrm{BrS}_{2}[\mathrm{M}+\mathrm{H}]^{+}: 470.1500$; found: 470.1500 .

t-Hexamer-3. Using a modified literature procedure ${ }^{52}$ an oven dried flask, equipped with a reflux condenser, was charged with Mg turnings $(0.115 \mathrm{~g}, 4.70 \mathrm{mmol})$ and diethyl ether $(10 \mathrm{~mL})$ and was brought to gentle reflux. To this solution was added dropwise via syringe, a solution of 5'-bromo-5-(2-hexyldecyl)-2,2'bithiophene $(0.213 \mathrm{~g}, 0.450 \mathrm{mmol})$ in diethyl ether $(1 \mathrm{~mL})$. Two drops of 1,2-dibromoethane were added, and the reaction mixture was heated at reflux for $1 \mathrm{~h}$. The Grignard solution was stirred for another hour at ambient temperature, and was transferred dropwise to a second reaction vessel containing a refluxing solution of $5,5^{\prime}$-dibromo-2, $2^{\prime}$-bithiophene $(0.0500 \mathrm{~g}$, $0.150 \mathrm{mmol})$ and $\mathrm{Ni}(\mathrm{dppp}) \mathrm{Cl}_{2}(0.0030 \mathrm{~g}, 0.0050 \mathrm{mmol})$ in toluene $(6 \mathrm{~mL})$. The resulting red-brown mixture was heated at reflux overnight and was then cooled and quenched with $6 \mathrm{~N} \mathrm{HCl}$ $(2 \mathrm{~mL})$. The organic phase was washed with water and brine, was dried $\left(\mathrm{Na}_{2} \mathrm{SO}_{4}\right)$, and the solvent was removed in vacuo. The crude mixture was purified by column chromatography (silica gel; hexanes, then $3: 1 \mathrm{v} / \mathrm{v}$ hexanes : ethyl acetate), followed by recrystallization from hexanes to afford the desired compound as a red-orange solid $(0.080 \mathrm{~g}, 34 \%$ yield $) .{ }^{1} \mathrm{H} \mathrm{NMR}\left(\mathrm{CDCl}_{3}\right.$, $400 \mathrm{MHz}): \delta 0.86(\mathrm{t}, J=8.0 \mathrm{~Hz}, 12 \mathrm{H}), 1.25-1.28(\mathrm{br}, 48 \mathrm{H}), 1.62$ (br m, 1H), $2.71(\mathrm{~d}, J=6.4 \mathrm{~Hz}, 4 \mathrm{H}), 6.64(\mathrm{~d}, J=3.6,2 \mathrm{H}), 6.97(\mathrm{~d}$, $J=3.6,2 \mathrm{H}), 6.98(\mathrm{~d}, J=3.6,2 \mathrm{H}), 7.03-7.06(\mathrm{~m}, 6 \mathrm{H}) .{ }^{13} \mathrm{C} \mathrm{NMR}$ $\left(\mathrm{CDCl}_{3}, 100 \mathrm{MHz}\right): \delta 14.1,22.7,26.6,29.3,29.6,29.9,31.92$, 33.2, 34.6, 40.0, 123.4, 123.5, 124.1, 124.3, 126.0, 134.6, 135.0, $135.7,136.2,137.1,144.4$. HRMS $m / z$ calcd for $\mathrm{C}_{56} \mathrm{H}_{78} \mathrm{~S}_{6}[\mathrm{M}+$ $\mathrm{H}]^{+}$: 942.4428; found: 944.4422 .

t-Hexamer-2 and t-nonamer. A solution of 3,3"-dihexyl$2,2^{\prime}: 5^{\prime}, 2^{\prime \prime}$-terthiophene ${ }^{53}(1.21 \mathrm{~g}, 2.91 \mathrm{mmol})$ in THF $(45 \mathrm{~mL})$ was cooled to $-30{ }^{\circ} \mathrm{C}$. A solution of $n$-butyllithium $(1.16 \mathrm{~mL}, 2.5 \mathrm{M}$ in hexanes) was added dropwise over $5 \mathrm{~min}$, and the resulting solution was stirred at $-30{ }^{\circ} \mathrm{C}$ for $1 \mathrm{~h}$. The solution was then cooled further to $-70{ }^{\circ} \mathrm{C}$, and anhydrous copper(II) chloride $(0.468 \mathrm{~g}, 3.49 \mathrm{mmol})$ was added. The solution was then warmed to ambient temperature and was stirred overnight. The reaction was quenched through the addition of brine, and the solution was then extracted with diethyl ether. The combined extracts were washed with water and brine and were dried $\left(\mathrm{Na}_{2} \mathrm{SO}_{4}\right)$ and the solvent was removed in vacuo. The crude material was then purified by column chromatography (silica gel; hexanes, then 5\% dichloromethane/hexanes) to give t-hexamer-2 as an orange solid $(0.457 \mathrm{~g}, 38 \%)$ and $\mathbf{t}$-nonamer as a red solid $(0.020 \mathrm{~g}, 2 \%)$. The NMR data matched that previously reported in the literature. ${ }^{54}$

\subsection{Electrochemical and photophysical investigations}

Absorbance and fluorescence measurements were carried out in THF. The photoluminescence (PL) quantum yields $\left(\Phi_{\text {fluor }}\right)$ were obtained using fluorescein $\left(\Phi_{\text {fluor }}=0.95\right)$ as a standard. ${ }^{55}$ Absorbance measurements were recorded using a DU 640 spectrophotometer (Beckman, Fullerton, CA) and a $1 \mathrm{~cm}$ quartz cuvette. Fluorescence experiments were performed with a double-beam QuantaMaster Spectrofluorimeter (Photon Technology International, Birmingham, NJ). A $70 \mathrm{~W}$ Xe lamp was used as the light source and the slit width was set at $0.5 \mathrm{~mm}$. Electrochemical experiments were carried out in an argon atmosphere drybox (Vacuum Atmospheres Corp., Hawthorne, CA or UniLab 2000, M. Braun Inc. USA, Stratham, NH) to preclude the presence of oxygen and water. Solutions prepared in the drybox were tightly sealed with a Teflon cap. Prior to sealing, the absence of trace quantities of oxygen or other impurities was confirmed by $\mathrm{CV}$ inside the drybox. A $0.0314 \mathrm{~cm}^{2}$ platinum working electrode was used for the $\mathrm{CV}$ experiments and a right angle bent (J-type) Pt electrode with the same area was used for the ECL experiments involving a CCD. The geometric electrode area was determined by chronoamperometry using a $2 \mathrm{mM}$ solution of ferrocene in $\mathrm{MeCN}$. The diffusion coefficients of the various oligomers were obtained from the observed scan rate dependences based on the Randles-Ševčik equation and by chronoamperometric pulsing for $1 \mathrm{~s}$, using the Cottrell equation. Prior to each experiment the platinum working electrode was polished with $0.3 \mu \mathrm{m}$ alumina (Buehler, Ltd., Lake Bluff, IL), sonicated in acetone, ethanol and in water for $5 \mathrm{~min}$ and dried in the oven at $120{ }^{\circ} \mathrm{C}$ for $30 \mathrm{~min}$. An $\mathrm{Ag}$ wire was used as a quasireference electrode and a $\mathrm{Pt}$ wire was used as a counter electrode. The electrochemical potential of the quasi-reference electrode was calibrated using the ferrocene/ferrocenium couple as a standard, taking $E^{\mathrm{o}}=0.342 \mathrm{~V}$ vs. aq. $\mathrm{SCE}^{56} \mathrm{CV}$ and chronoamperometry measurements were carried out with CHI 660 , CHI 900B and CHI 660D electrochemical workstations $(\mathrm{CH}$ Instruments, Austin, TX). ECL spectra were obtained by using either a CHI 660 or an Eco Chemie Autolab PGSTAT30 potentiostat (Utrecht, the Netherlands) applying potentials $80 \mathrm{mV}$ more positive and more negative than the peak potentials of the first electrochemical waves. ECL experiments with benzoyl peroxide were carried out by pulsing to $80 \mathrm{mV}$ negative of the first reduction peaks. ECL spectra were recorded with a Princeton Instruments Spec-10 CCD Camera (Trenton, NJ) with an Acton SpectraPr-150 monochromator (Acton, MA). The wavelength was calibrated with a $\mathrm{Hg} / \mathrm{AR}$ pen-ray lamp from Oriel (Stratford, CT). ECL intensity transients were recorded prior the ECL spectral measurements using a photomultiplier tube (PMT, Hamamatsu R4220, Japan). A Kepco (New York) high voltage 
power supply at $-750 \mathrm{~V}$ was applied to the PMT. An electrometer connected with the computer and PMT (Model 6517, Keithley Instruments Inc., Cleveland, $\mathrm{OH}$ ) was used to measure the PMT signal. Digital simulations of the CVs for monomer, dimer and trimer were carried out using the Digisim software package (Bioanalytical systems, West Lafayette, IN). ${ }^{57-60}$

\section{Results and discussion}

\subsection{Photophysical investigations}

$\mathrm{UV}-\mathrm{Vis}$ and fluorescence results for the thiophene and fluorene oligomers are shown in Fig. 1 and Fig. S1, S2 (ESI $\dagger$ ) and are summarized in Table 1. As these properties have already been widely investigated, ${ }^{61-66}$ only a brief summary of the results is presented for purposes of comparison to the electrochemical and ECL results.

For the thiophene oligomers, substantial red shifts in both absorbance and fluorescence are seen in progressing from bithiophene (t-dimers), terthiophene (t-trimers), quaterthiophene (t-tetramer) and finally sexithiophene (t-hexamers), as would be expected for a congruent series of systems characterized by increasing electron delocalization. The relative shifts were smaller as the chain length increased from 6 to 12 units. The growth of the first, shorter wavelength shoulder and a decrease in the longer wavelength peak could also be correlated with structural changes because of the importance of geometrical
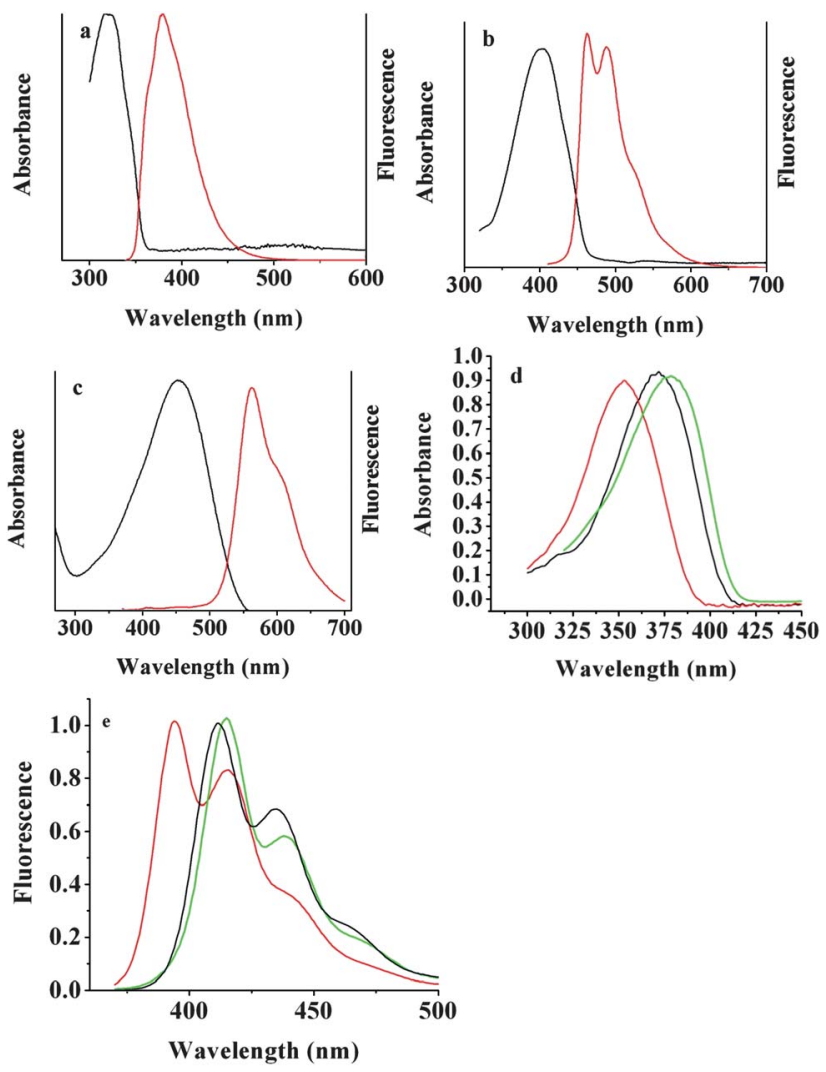

Fig. 1 Absorption (black) and fluorescence (red) spectra of $2 \mu \mathrm{M}$ thiophene dyes in DCM: (a) t-dimer; (b) t-tetramer; (c) t-dodecamer; (d) absorption and (e) fluorescence spectra of $2 \mu \mathrm{M}$ solution of the fluorenes: f-trimer (red); f-pentamer (black); f-heptamer (green). orientation. ${ }^{63}$ The presence of certain substituent groups may also influence the photophysical behavior. ${ }^{63}$ The fluorene oligomers showed a similar trend, with the shorter wavelength peak decreasing in intensity relative to that of the longer wavelength peak as the chain length increased. The presence of multiple peaks in the oligofluorene fluorescence spectrum has been ascribed to greater delocalization of the electron density in the excited state compared with the ground state, while a decrease in signal intensity for the longer wavelength spectral feature is thought to reflect a higher degree of conjugation. ${ }^{66}$

\subsection{Electrochemical studies}

The CV of the oligothiophenes and oligofluorenes are summarized in Table 2, Fig. 2 to 8, and Fig. S3 to S15†. For the sake of brevity, representative scan rate $(v)$ dependencies and digital simulations are mainly presented in the supporting information $\dagger$. Most previous studies of oligothiophenes have focused on oxidation, with fewer studies of reduction being reported in the literature. ${ }^{67}$ Presumably, this reflects the fact that the reductions in question typically occur at quite negative potentials and that oxidations are more frequently used in (p-type) doping of polythiophene polymers. One goal of the present study was thus to carry out analyses of a set of congeneric oligothiophenes under conditions of both oxidation and reduction.

The t-dimers show the presence of a single, one electron wave on both oxidation and reduction (Fig. 2a,b). The higher stability of t-dimer-2 is ascribed to the presence of the alkyl substitution in the $\alpha, \alpha^{\prime}$ positions. Our efforts to access the second electrochemical oxidation wave were limited by the solvent potential window, although reversible two-electron behavior was noted by Heinze et al. for a $\beta, \beta^{\prime}$-modified thiophene dimer at $-40^{\circ} \mathrm{C}$ using purified liquid sulfur dioxide as the medium. ${ }^{10}$ Instability upon oxidation has been ascribed in part to the absence of substituents in the $\beta$-position, at least in the case of small oligothiophenes. ${ }^{40,41}$ However, two oxidation and reduction waves can be seen for both t-trimer-1 and t-trimer-2, with chemical reversibility being noted for both the first and second waves (Fig. 2c-f). Using $1: 1$ $(\mathrm{v} / \mathrm{v})$ benzene : $\mathrm{MeCN}$ as the solvent, one can obtain a fairly wide range of potentials $(\sim+2.2$ to -2.6$)$ for both oxidation and reduction and this is useful in comparing the electrochemical spacing between the first oxidation and first reduction processes and hence assigning the observed spectroscopic transition to the first electronically excited state. ${ }^{68}$ This stands in contrast to what has been seen in studies involving different solvents for the oxidation and reduction (e.g., dichloromethane and THF, respectively) and highlights the fact that unknown solvent effects can introduce uncertainties into the measured potentials. These effects are caused by interaction of the produced ions or radical ions with certain solvents and formation of different reaction products. Electrochemical studies of some substituted oligomers were carried out by Hapiot et al., but those studies are mostly concerned with oxidation and its importance for oligomerization without paying too much attention to reduction or withdrawing of multiple electrons on oxidation. ${ }^{15,69}$

The use of MeCN as the electrochemical solvent was possible only with the heavily alkylated t-trimer-2, due to the limited solubility of the other oligomers studied. Both t-trimers show similar electrochemical behavior. Specifically, chemically 
Table 1 Photophysical properties of the thiophene ( $t$ ) and fluorene (f) dyes

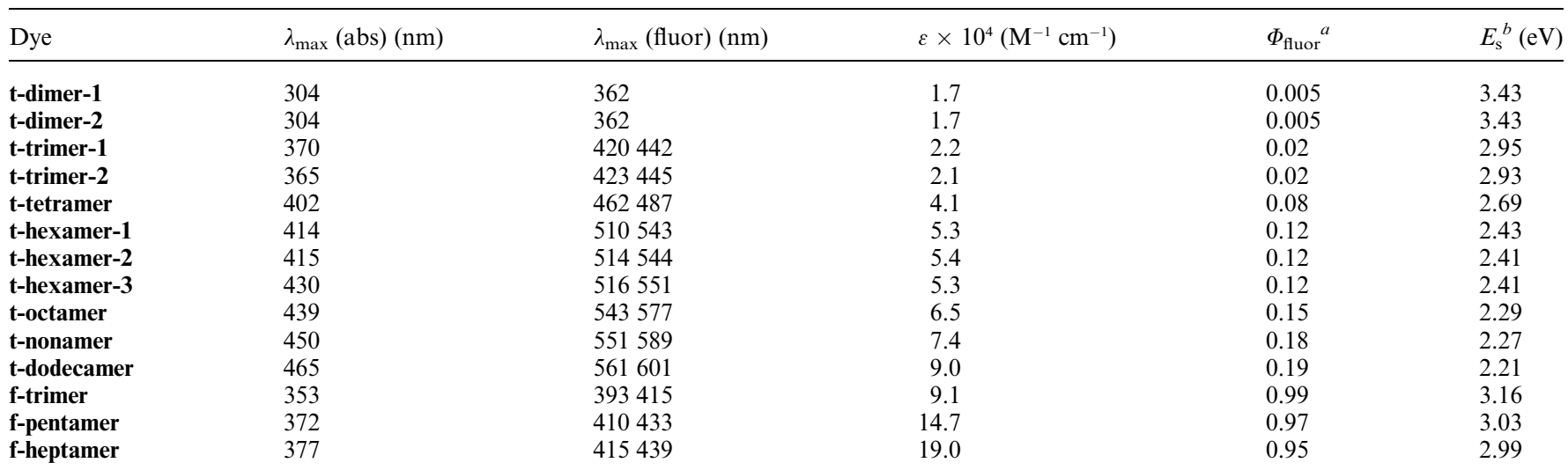

${ }^{a}$ Relative to fluorescence of fluorescein. ${ }^{b} E_{\mathrm{s}}$ - approximate energy of the singlet state taken as the fluorescence wavelength maximum. First peaks were used for calculations.

reversible first waves are seen for both systems. In addition, some instability involving the second oxidation and reduction waves was seen in both cases.

The quaterthiophene t-tetramer is characterized by the presence of two nernstian first waves on both oxidation and reduction with instability in the second reduction process being seen that is ascribed to limitations involving the solvent window (Fig. 2g,h). The CV of t-hexamer-1 shows the presence of only a small signal due to the low solubility of this compound (Fig. 3a). t-hexamer-2, with unblocked $\alpha, \alpha^{\prime}$-positions, shows the presence of two reversible waves on reduction and instability upon oxidation (Fig. 3b). Conversely, t-hexamer-3, which features long, branched alkyl chains in the $\alpha$-positions, is characterized by the presence of two reversible waves on both oxidation and reduction (Fig. 3c). Consistent with what is seen for t-hexamer-2, the CVs of the sterically unhindered t-octamer, t-nonamer and t-dodecamer reveal the presence of features that are characteristic of irreversibility (Fig. 3d-f). This can be caused by instability of the radical anion when reaching the negative limit of the solvent window. Despite this, the appearance of multiple waves is clear. Scanning to further positive and negative potentials for the t-hexamer-3 and to negative potentials in the case of t-hexamer-2 reveals the presence of the third electrochemical waves (Fig. $4 \mathrm{a}-\mathrm{c})$. These waves occur at approximately $-2.4 /-2.5 \mathrm{~V}$ for reduction of both dyes and 1.9/2.0 $\mathrm{V}$ for the oxidation of t-hexamer-3 (Fig. $4 \mathrm{a}-\mathrm{c}$ ).

The phenomenon of multiple electron transfer events is more obvious in the case of the higher oligomers t-octamer, t-nonamer

Table 2 Electrochemical and ECL properties of the oligothiophene and oligofluorene dyes

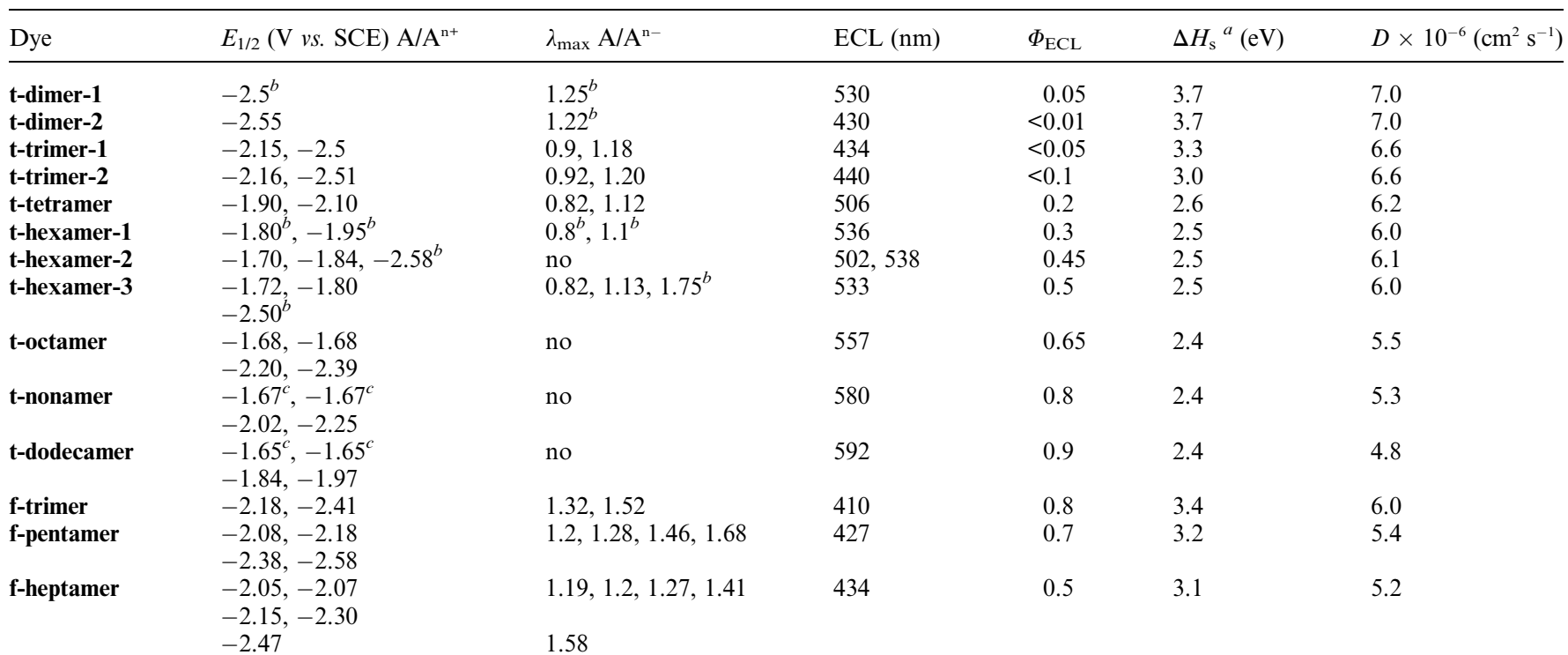

${ }^{a} \Delta H_{\mathrm{s}}$ is energy of the singlet state calculated assuming presence of the entropy factor of $0.1 \mathrm{~V}$; in case of irreversible behavior values close to the beginning of the electrochemical waves were used. ${ }^{b}$ The potential close to the peak was taken for oxidation, for all other values the numbers were obtained from digital simulations. ${ }^{c}$ Simultaneous electron transfer. ECL quantum yield $(\Phi)$ was determined $v s$. Ru(bpy) ${ }_{3}{ }^{2+}$. 

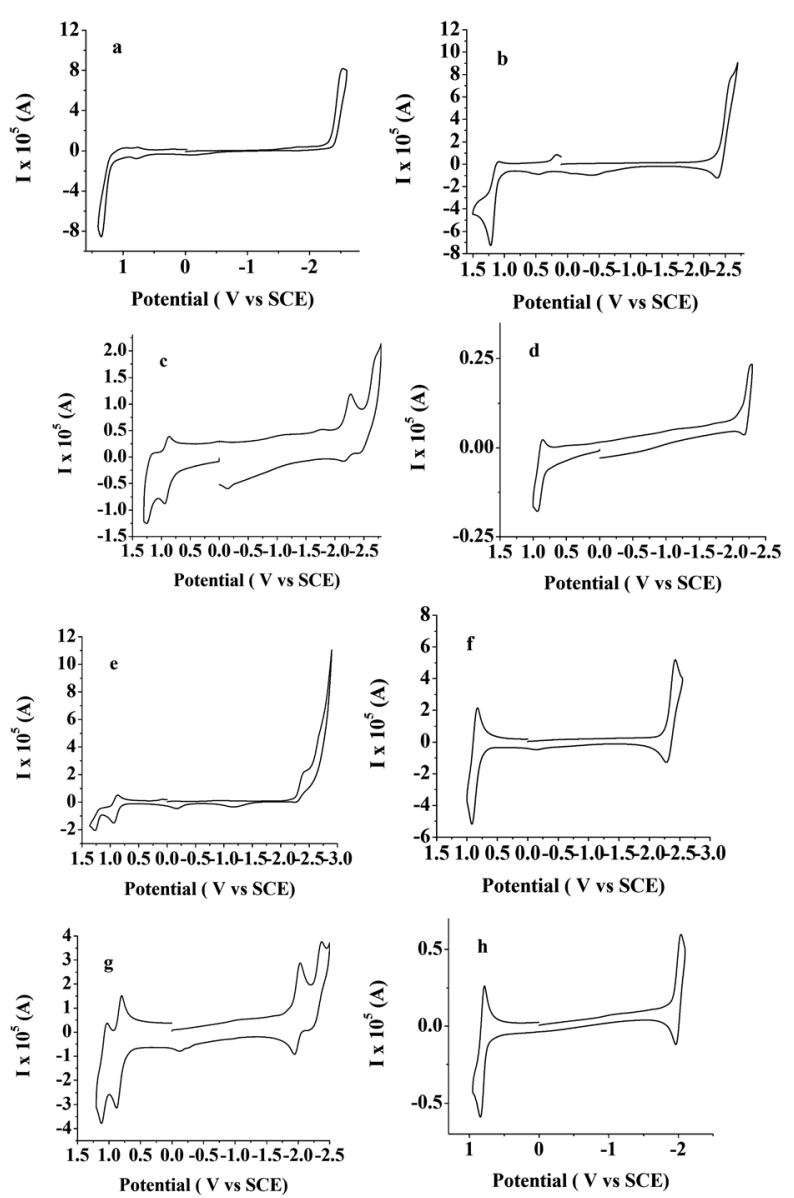

Fig. 2 Cyclic voltammograms of (a) $4.0 \mathrm{mM}$ t-dimer-1; (b) $4.2 \mathrm{mM}$ tdimer-2; (c) $2.0 \mathrm{mM}$ and (d) $0.3 \mathrm{mM}$ t-trimer-1; (e) and (f) $1.9 \mathrm{mM} \mathrm{t}$ trimer-2; (g) and (h) $0.8 \mathrm{mM}$ t-tetramer. Scan rate: $0.1 \mathrm{~V} \mathrm{~s}^{-1}$, except (b) and (f), where $1 \mathrm{~V} \mathrm{~s}^{-1}$ was used and $(\mathrm{g})$ where $2 \mathrm{~V} \mathrm{~s}^{-1}$ was used. Solvent: $1: 1$ benzene : acetonitrile; supporting electrolyte: $0.1 \mathrm{M} \mathrm{TBAPF}_{6}$; platinum electrode area: $0.0314 \mathrm{~cm}^{2}$.

and t-dodecamer (Fig. 4d-f). In this case, digital simulations confirm presence of multiple electron transfer events (Fig. S10$\mathrm{S} 12 \dagger)$.

In general, the oligofluorenes showed a larger separation between oxidation and reduction peaks, around 3.3-3.5 V, with a slight decrease in the case of transition from trimer to heptamer (Fig. 5a-c). For example, two waves are observed upon both oxidation and reduction for the f-trimer (Fig. 5a, 6a-b, S13†). Presumably, this reflects limitations imposed by the solvent window. Consistent with this conclusion is the fact that both oxidation and reduction are observed as multiple electron waves in the case of the f-pentamer and f-heptamer (Fig. 5b-c, 6, S14 $\mathrm{S} 15 \dagger)$.

A good correlation between electrochemical and photophysical properties is observed for the systems studied. Specifically, the singlet state energies as determined spectroscopically correlate well with the potential spacings between the first oxidation and reduction waves (Table 2).

The hexyl chains on the oligofluorenes are thought to afford better blocking of the reactive sites presumably generated in the oxidized species as compared to the corresponding
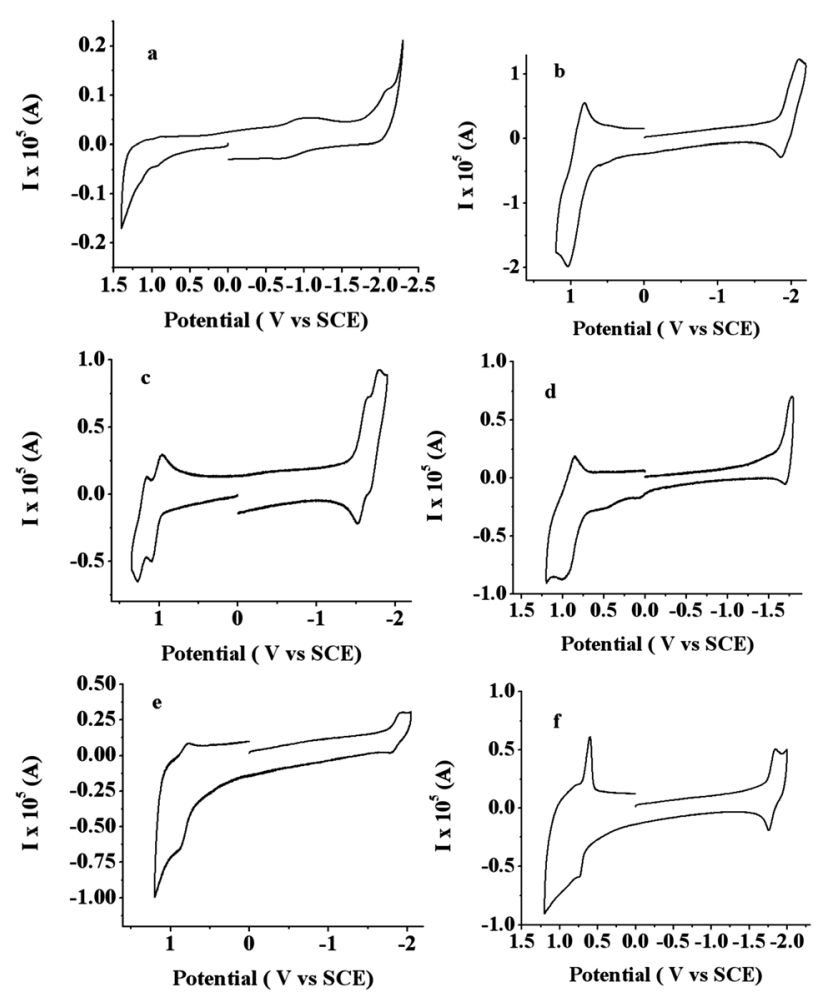

Fig. 3 Cyclic voltammograms of (a) $0.04 \mathrm{mM}$ t-hexamer-1; (b) $0.3 \mathrm{mM}$ thexamer-2; (c) $0.3 \mathrm{mM}$ t-hexamer-3; (d) $0.18 \mathrm{mM}$ t-octamer; (e) $0.1 \mathrm{mM}$ tnonamer; (f) $0.2 \mathrm{mM}$ t-dodecamer; scan rate: $1 \mathrm{~V} \mathrm{~s}^{-1}$; solvent: $1: 1$ benzene : acetonitrile; supporting electrolyte: $0.1 \mathrm{M} \mathrm{TBAPF}$; platinum electrode area: $0.0314 \mathrm{~cm}^{2}$.

oligothiophenes. This results in a more stable oxidation process, with stability of the f-trimer being similar to that of the $\mathbf{t}$ dodecamer. The fluorene trimers and the thiophene dodecamers are not prone to oxidative oligomerization (Fig. 7a,b). This is true even over the course of multiple $\mathrm{CV}$ scans, where no evidence of polymer peaks is observed. However scans to more positive potentials promote oligomerization. This finding is consistent with the greater reactivity expected for the more highly oxidized species (Fig. 7c,d). Formation of the polymer or dimer with further formation of the adsorbed layer is also possible in this case. ${ }^{19}$

\subsection{Discussion of the electrochemical studies}

The appearance of discrete separate waves in the CVs of the oligomers permits the assignment of some guidelines for the addition or loss of electrons from different monomer units within a related set of oligomers (Fig. 8). Ultimately, such structureproperty studies are expected to be useful in understanding the properties of conjugated polymers containing the same building blocks as are present in the oligothiophenes and oligofluorenes of the present study. ${ }^{70-73}$ Some of these guidelines were already proposed by Heinze et al. for phenylenes and phenylvinylene oligomers and oligoenes, ${ }^{18,19}$ and have been complemented by various theoretical treatments, e.g. VEH calculations of the first ionization potential and the electron affinities of thiophene oligomers and other monomers as a function of size. ${ }^{74}$ Heinze 

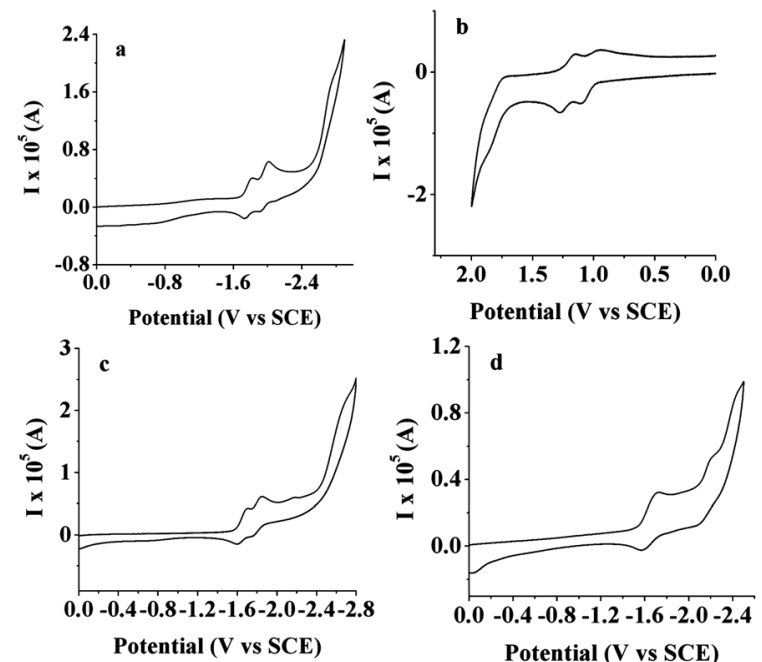

Potential (V vs SCE)
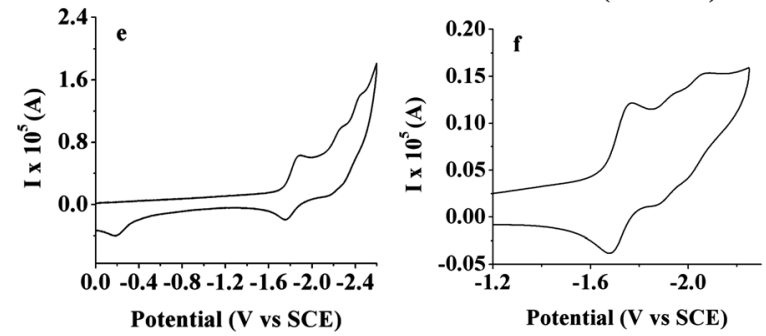

Fig. 4 Cyclic voltammograms of (a) and (b) $0.6 \mathrm{mM}$ t-hexamer-2; (c) $0.6 \mathrm{mM}$ t-hexamer-3; (d) $0.18 \mathrm{mM}$ t-octamer; (e) $0.4 \mathrm{mM}$ t-nonamer; (f) $0.15 \mathrm{mM}$ t-dodecamer. Scan rate: $0.1 \mathrm{~V} \mathrm{~s}^{-1}$; supporting electrolyte: $0.1 \mathrm{M}$ $\mathrm{TBAPF}_{6}$; platinum electrode area: $0.0314 \mathrm{~cm}^{2}$.
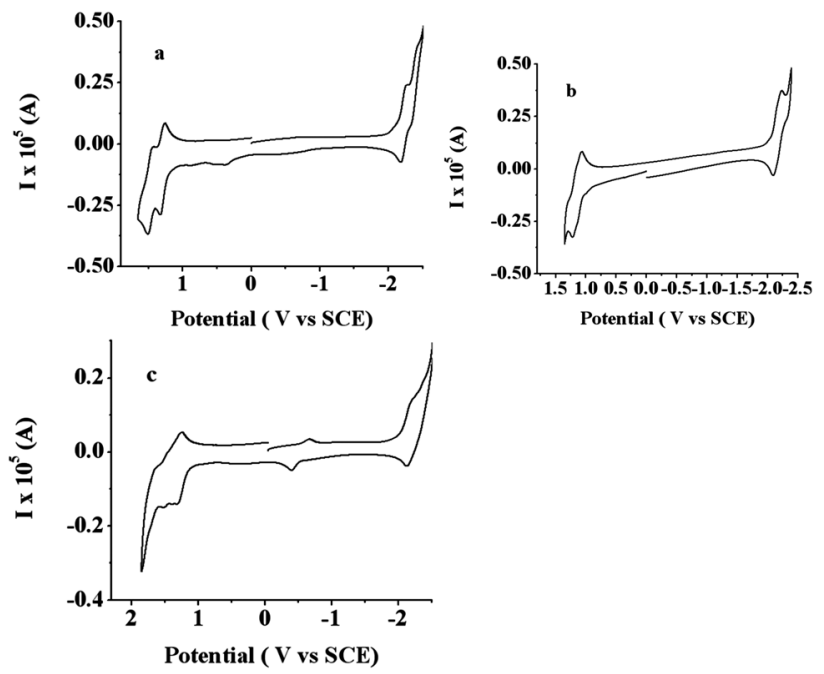

Fig. 5 Cyclic voltammograms of (a) $0.3 \mathrm{mM}$ f-trimer; (b) $0.25 \mathrm{mM}$ fpentamer; (c) $0.14 \mathrm{mM}$ f-heptamer; scan rate: $0.1 \mathrm{~V} \mathrm{~s}^{-1}$; solvent: $1: 1$ benzene : acetonitrile; supporting electrolyte: $0.1 \mathrm{M} \mathrm{TBAPF}_{6}$; platinum electrode area: $0.0314 \mathrm{~cm}^{2}$.

et al. have demonstrated a shift of the electrochemical potential for both oxidation and reduction to lower potentials with an increase in chain length and a decrease of the separation between electrochemical waves because of strong coulombic interactions. ${ }^{17-19}$ For our systems, in general, the addition of the first electron to a thiophene oligomer and both addition and removal
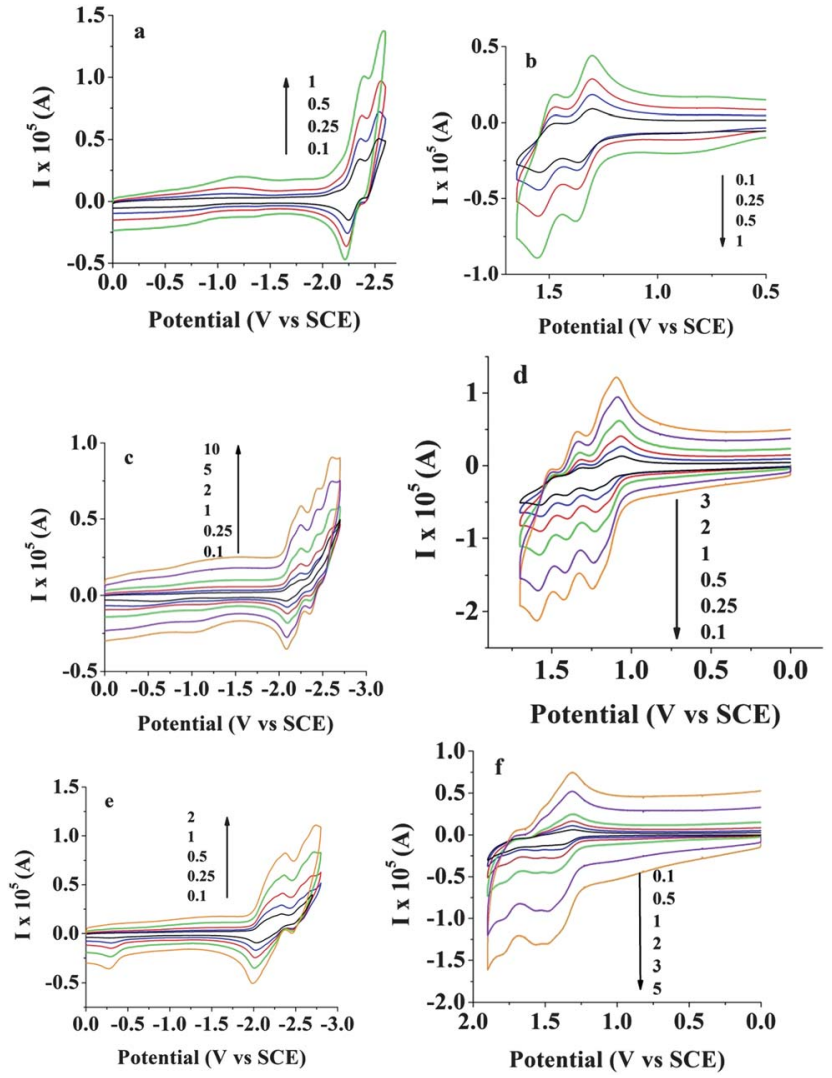

Fig. 6 Scan rate dependence for fluorene oligomers; (a) and (b) $0.3 \mathrm{mM}$ f-trimer; (c) and (d) $0.08 \mathrm{mM}$ f-pentamer; (e) and (f) $0.14 \mathrm{mM}$ f-heptamer. Arrows show direction of the scan rate in $\mathrm{V} \mathrm{s}^{-1}$. Experimental data: solvent: (a,c,e) THF, (b,d,f) $1: 1$ benzene : acetonitrile; supporting electrolyte: $0.1 \mathrm{M} \mathrm{TBAPF}_{6}$; platinum electrode area: $0.0314 \mathrm{~cm}^{2}$.
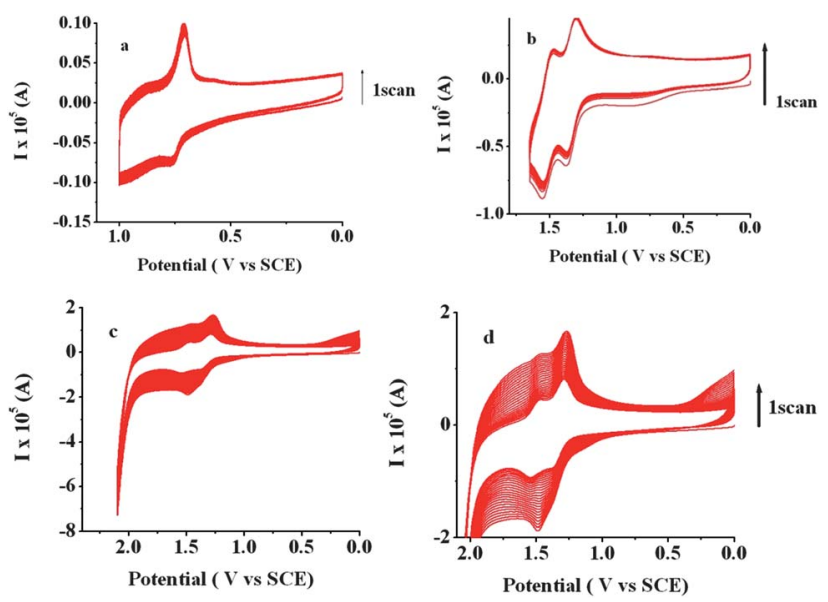

Fig. 7 Multiple scan rate experiment for (a) $0.08 \mathrm{mM}$ t-dodecamer and (b-d) $0.3 \mathrm{mM}$ f-fluorene. Experimental data: scan rate $0.25 \mathrm{~V} \mathrm{~s}^{-1}$ for (a) and $1 \mathrm{~V} \mathrm{~s}^{-1}$ for (b-d); solvent: $1: 1$ benzene : acetonitrile; supporting electrolyte: $0.1 \mathrm{M} \mathrm{TBAPF}_{6}$; platinum electrode area: $0.0314 \mathrm{~cm}^{2}$.

of the first electron from a fluorene oligomer becomes easier as the size increases. This can be seen by inspection of Fig. 8. For longer oligomers $(n=8-12)$ (Fig. $8 \mathrm{a}$ and $8 \mathrm{~b})$, the interaction energy involving a distant monomer unit is so small, that the potential for the addition of the second electron occurs at the 

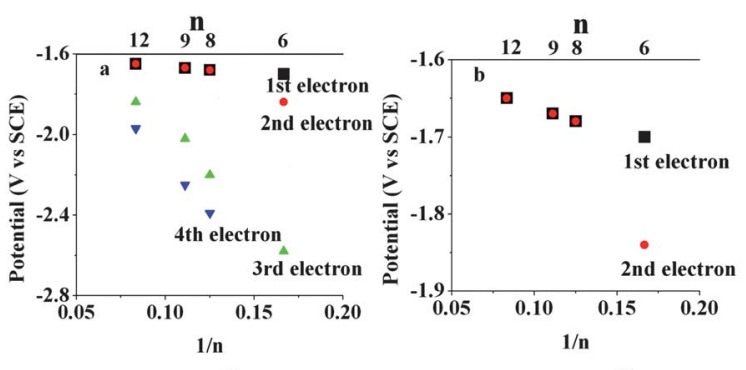

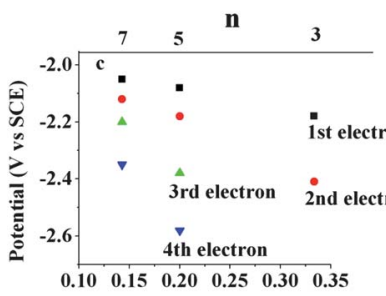

$1 / \mathbf{n}$

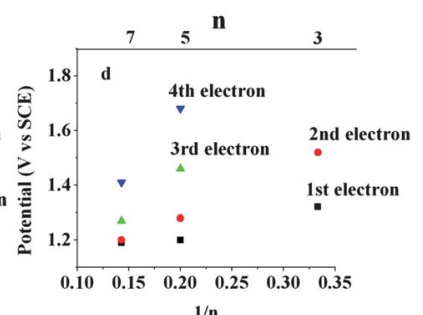

$1 / \mathbf{n}$
Fig. 8 Potential dependence on $1 / n$ for the t-thiophenes dyes $(a-b)$ and (c-d) f-fluorenes dyes. The colored symbols are correlated with addition of consecutive electrons: black squares (first electron); red circles ( 2 electron); green triangles up (3 electron); and blue triangles down (4 electron); $n=6,8,9,12$ are corresponded with t-hexamer-2, t-octamer, t-nonamer, t-dodecamer and $\boldsymbol{n}=\mathbf{3 , 5}, \mathbf{7}$ are corresponded with f-trimer, f-pentamer and f-heptamer.

same microscopic potential as the first. An additional conclusion is that it is relatively harder (larger $\Delta E$ ) to remove or add the third electron compared to the second, as seen, for example, in the larger $\Delta E_{2,3}$ separations for the f-pentamer and $\mathbf{t}$-hexamer as compared with the corresponding $\Delta E_{1,2}$ values. The same trend was seen in other examples involving phenylvinylenes, thiophenes, and BODIPY oligomers. ${ }^{11,19,75}$

The energetic separation for addition of the second electron relative to the first varies with the extent of conjugation and the size of the oligomer. For example the thiophene trimer shows a separation between first and second electron reduction waves $\left(\Delta E_{1,2}\right)$ of around $0.4 \mathrm{~V}$, which is larger than the $0.2 \mathrm{~V}$ for the corresponding oligofluorenes and $0.1 \mathrm{~V}$ seen for typical BODIPY dyes. ${ }^{11,38}$ This trend was also noticed for the bicyclo[2,2,2]octene segregated thiophene oligomers and 3,4-propylenedioxythiophene, where the appearance of a third wave was seen in the case of the hexamer..$^{75,76}$ The fact that adding a third electron is energetically much more difficult than adding either of the first two electrons can be understood in terms of repulsive coulombic forces. For example, upon addition of electrons to a hexamer, one can envision that addition of a second electron will result in increased electron densities at the extreme ends of the polymers (units 1 and 6). Addition of the next electron should thus occur at a position of minimum coulombic repulsion, e.g. at subunits 3 or 4 , and will be subject to a larger repulsive force.

At large $n$ (number of repeating units), the limit of conjugation is reached and no further substantial change in the electrochemical potential with $n$ is observed for the 1 st electron transfer (Fig. $8 \mathrm{a}-\mathrm{d}$ ). The difference between the first oxidation and reduction potentials, corresponding to the difference between the LUMO and the HOMO, corresponds to the "electronic energy gap" of the molecule and relates to the ECL behavior discussed below. Second and consecutive peaks reflect interactions between different monomer units. This addition is correlated with a steeper curve in plots of the dependence of the electrochemical potential on $1 / n$ than for the corresponding first electron transfer. With increasing molecule size the absence of substantial interactions eventually permits the concurrent and simultaneous addition of electrons.

Summarizing this section, all of the results show the importance of both electrostatic interactions and electronic delocalization on the magnitude of the potential difference between electrochemical waves, where $\Delta E_{1,2}<\Delta E_{2,3}$ for both systems. This important feature for oligomeric systems can explain the difficulty of experimentally obtaining third oxidation and reduction for some systems within the solvent/electrolyte window. This can lead to questionable conclusions about the formation of only doubly charged species and the absence of the further electrochemical processes with polymers (Fig. 5a, 6a-b, S12 $\dagger$ ), especially in the presence of ionic strength and ion pairing effects.

\subsection{Electrogenerated chemiluminescence}

ECL spectra were obtained for all of the compounds investigated. Several studies on the ECL behavior of some thiophene and spirofluorene oligomers have been previously reported. ${ }^{38,67,77-80}$ In this work the ECL spectra for oligomers with a large number of units, $n$, were obtained. In terms of length, this corresponds to a maximum $n$ value of seven in the case of the fluorenes and twelve in the case of the thiophenes.

Annihilation ECL, which corresponds with production of the radical ions can be presented as:

$$
\begin{gathered}
\mathrm{A}+\mathrm{e}^{-} \rightarrow \mathrm{A}^{-} \\
\mathrm{A}-\mathrm{e}^{-} \rightarrow \mathrm{A}^{+} \\
\mathrm{A}^{-}+\mathrm{A}^{+} \rightarrow \mathrm{A}^{*} \\
\mathrm{~A}^{*} \rightarrow \mathrm{A}+\mathrm{h} v
\end{gathered}
$$

Representative results are given in the main text with additional results in the supporting information (Fig. 9-10, Fig. S16†). Thiophene t-dimer-1 does not show an ECL signal in the absence of coreactant as a consequence of its instability upon oxidation and reduction. Use of benzoyl peroxide as a coreactant with the reduction of unblocked $\mathbf{t}$-dimer-1 causes the appearance of a strong ECL signal with a maximum wavelength of emission at $530 \mathrm{~nm}$, which is very different from fluorescence wavelength at $360 \mathrm{~nm}$ (Fig. 9). Addition of benzoyl peroxide does not cause chemical reaction of the dimer in the absence of any redox process over an extended time, demonstrating that an electrochemical reduction process causes formation of the tetramer or larger oligomers. Formation of the electrochemical products monitored by ECL has been reported earlier for the reductive dimerization of fluoranthene in the presence of persulfate and for the unblocked BODIPY dyes. ${ }^{43,44}$ The mechanism of the activity for benzoyl peroxide ECL can take place as follows:

$$
\begin{gathered}
\mathrm{HA}-\mathrm{AH}+\mathrm{e} \rightarrow \mathrm{HA}^{-} \mathrm{AH}^{-} \\
\mathrm{BPO}+\mathrm{e} \rightarrow \mathrm{BPO}^{-} \\
\mathrm{BPO}^{-} \rightarrow \mathrm{C}_{6} \mathrm{H}_{5} \mathrm{CO}_{2}^{-}+\mathrm{C}_{6} \mathrm{H}_{5} \mathrm{CO}_{2}^{-}
\end{gathered}
$$




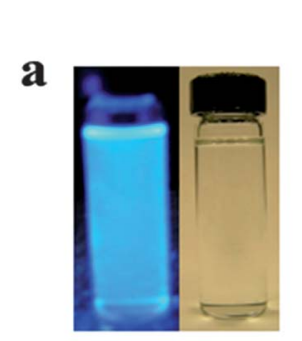

b

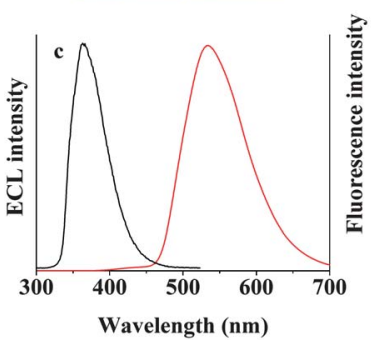

Fig. 9 Schematic representation of the fluorescence and ECL data for the t-dimer-1: (a) and (b) UV and fluorescence images for the thiophene tdimer-1 in the presence and absence of the benzoyl peroxide; third image from the right in (b) is an ECL image of the thiophene dimer; (c) ECL (red line) and fluorescence (black line) spectra for the $2.5 \mathrm{mM}$ t-dimer-1 in the presence of $10 \mathrm{mM}$ benzoyl peroxide. ECL spectrum was generated by cycling $80 \mathrm{mV}$ from the reduction peaks; supporting electrolyte: $0.1 \mathrm{M}$ TBAPF $_{6}$, platinum electrode area: $0.0314 \mathrm{~cm}^{2}$.

$$
\begin{gathered}
\mathrm{C}_{6} \mathrm{H}_{5} \mathrm{CO}_{2}+\mathrm{HA}-\mathrm{AH} \rightarrow \mathrm{HA}-\mathrm{AH}^{+}+\mathrm{C}_{6} \mathrm{H}_{5} \mathrm{CO}_{2}{ }^{-} \\
\mathrm{HA}-\mathrm{AH}^{+}+\mathrm{HA}-\mathrm{AH} \rightarrow \mathrm{HA}-\mathrm{A}-\mathrm{A}-\mathrm{AH}^{+} \\
\mathrm{HA}-\mathrm{A}-\mathrm{A}-\mathrm{AH}^{+}+\mathrm{HA}-\mathrm{AH} \rightarrow \mathrm{HA}-\mathrm{A}-\mathrm{A}-\mathrm{A}-\mathrm{A}-\mathrm{AH}^{+} \\
\mathrm{HA}-\mathrm{A}-\mathrm{A}-\mathrm{A}-\mathrm{A}-\mathrm{AH}^{+} \rightarrow \mathrm{HA}-\mathrm{A}-\mathrm{A}-\mathrm{A}-\mathrm{A}-\mathrm{AH}+\mathrm{H}^{+} \\
\mathrm{HA}-\mathrm{A}-\mathrm{A}-\mathrm{A}-\mathrm{A}-\mathrm{AH}+\mathrm{e} \rightarrow \mathrm{HA}-\mathrm{A}-\mathrm{A}-\mathrm{A}-\mathrm{A}-\mathrm{AH}^{-} \\
\mathrm{HA}-\mathrm{A}-\mathrm{A}-\mathrm{A}-\mathrm{A}-\mathrm{AH}{ }^{+}+\mathrm{HA}-\mathrm{A}-\mathrm{A}-\mathrm{A}-\mathrm{A}-\mathrm{AH}{ }^{-} \rightarrow \\
\mathrm{HA}-\mathrm{A}-\mathrm{A}-\mathrm{A}-\mathrm{A}-\mathrm{AH}{ }^{*}+\mathrm{HA}-\mathrm{A}-\mathrm{A}-\mathrm{A}-\mathrm{A}-\mathrm{AH} \\
\mathrm{HA}-\mathrm{A}-\mathrm{A}-\mathrm{A}-\mathrm{A}-\mathrm{AH}{ }^{*} \rightarrow \mathrm{HA}-\mathrm{A}-\mathrm{A}-\mathrm{A}-\mathrm{A}-\mathrm{AH}+\mathrm{h} v
\end{gathered}
$$

This mechanism is radical-substrate coupling (rsc). ${ }^{81}$ The other mechanism including radical-radical coupling ( $\mathrm{rrc}$ ) is also possible: ${ }^{11,81}$

$$
\begin{gathered}
\mathrm{HA}-\mathrm{AH}^{+}+\mathrm{HA}-\mathrm{AH}^{+} \rightarrow \mathrm{HA}-\mathrm{A}-\mathrm{A}-\mathrm{AH}^{2+} \\
\mathrm{HA}-\mathrm{A}-\mathrm{A}-\mathrm{AH}^{2+} \rightarrow \mathrm{HA}-\mathrm{A}-\mathrm{A}-\mathrm{AH}+2 \mathrm{H}^{+} \\
\mathrm{C}_{6} \mathrm{H}_{5} \mathrm{CO}_{2}+\mathrm{HA}-\mathrm{A}-\mathrm{A}-\mathrm{AH} \rightarrow \mathrm{HA}-\mathrm{A}-\mathrm{A}-\mathrm{AH}^{+}+\mathrm{C}_{6} \mathrm{H}_{5} \mathrm{CO}_{2}^{-} \\
\mathrm{HA}-\mathrm{A}-\mathrm{A}-\mathrm{AH}^{+}+\mathrm{HA}-\mathrm{AH}^{+} \rightarrow \mathrm{HA}-\mathrm{A}-\mathrm{A}-\mathrm{A}-\mathrm{A}-\mathrm{AH}+2 \mathrm{H}^{+} \\
\mathrm{HA}-\mathrm{AH}{ }^{-}+\mathrm{HA}-\mathrm{A}-\mathrm{A}-\mathrm{A}-\mathrm{A}-\mathrm{AH} H^{+} \rightarrow \\
\mathrm{HA}-\mathrm{A}-\mathrm{A}-\mathrm{A}-\mathrm{A}-\mathrm{AH}^{*}+\mathrm{HA}-\mathrm{AH}
\end{gathered}
$$

While methods exist for distinguishing between the rsc and rrc routes in typical electrochemical systems involving radical cation generation, ${ }^{82}$ the exact mechanism of the dimerization and
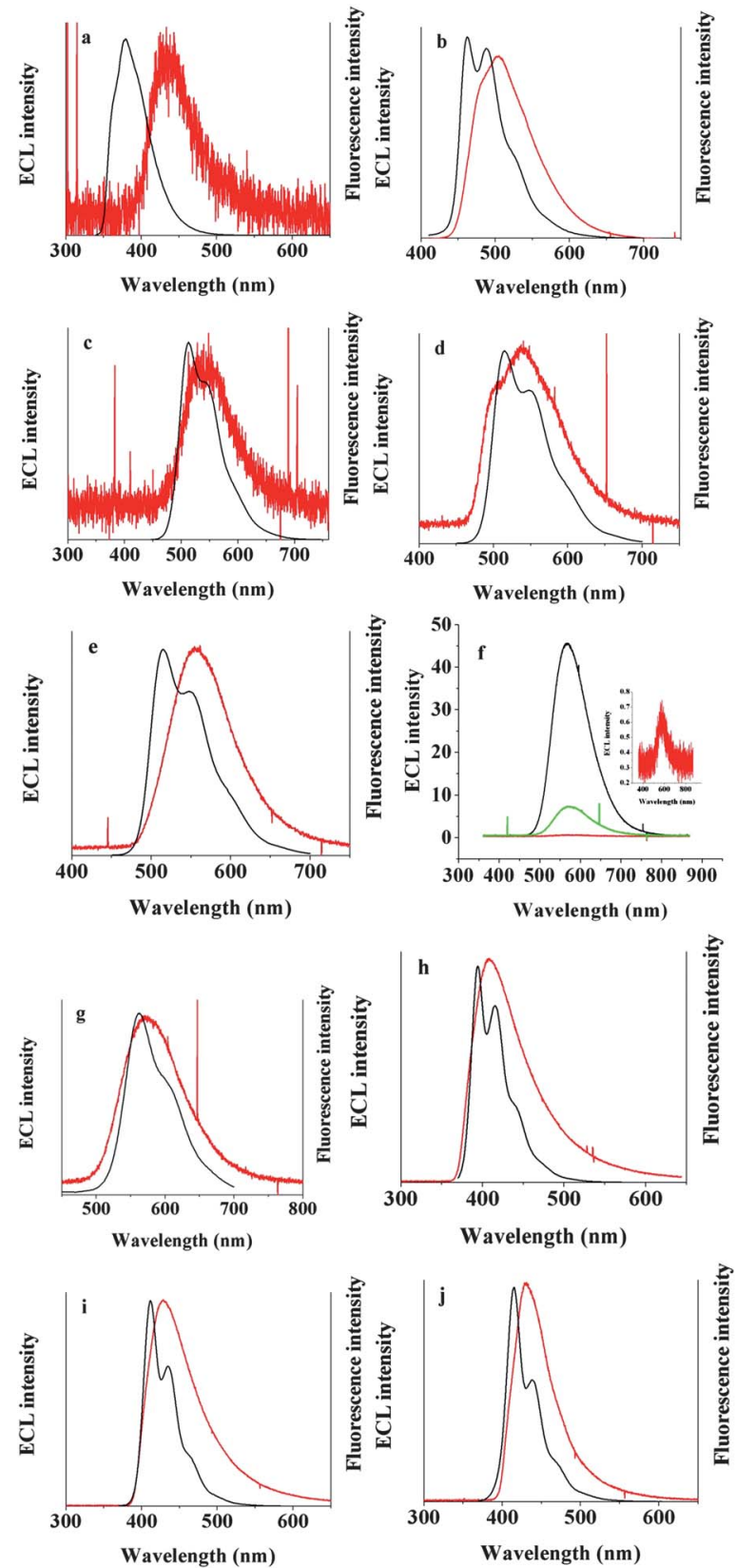

Fig. 10 Electrogenerated chemiluminescence spectra of (a) $4.2 \mathrm{mM} \mathrm{t}$ dimer-2; (b) $0.5 \mathrm{mM}$ solution of t-tetramer; (c) $0.15 \mathrm{mM}$ solution of thexamer-1; (d) and (e) $0.15 \mathrm{mM}$ t-hexamer-3; (f) comparison spectra of 20 $\mathrm{nM}, 2 \mathrm{nM}$ and $20 \mathrm{pM}$ of the t-dodecamer; $(\mathrm{g})$ comparison spectra of the fluorescence spectra of a $2 \mu \mathrm{M}$ solution with the ECL spectra of a $20 \mathrm{nM}$ solution of the t-dodecamer; (h-j) ECL spectra for a $0.5 \mathrm{mM}$ solution of (h) f-trimer, (i) f-pentamer, (j) f-heptamer; spectra (a)-(d), (h)-(j) were obtained by annihilation from $80 \mathrm{mV}$ from the first peaks. All other spectra were obtained by using $10 \mathrm{mM}$ benzoyl peroxide and applying potential $80 \mathrm{mV}$ negative from the first reduction wave; solvent: $1: 1$ benzene : acetonitrile; supporting electrolyte: $0.1 \mathrm{M} \mathrm{TBAPF}_{6}$; platinum electrode area: $0.0314 \mathrm{~cm}^{2}$.

further oligomerization is difficult to determine in the present case because of the presence and reactivity of the coreactant, BPO. Generally the rrc mechanism is found in most studies for the dimerization or oligomerization of monomers or short oligomers. ${ }^{6,11}$ 
This mechanism differs from that usually seen for ECL, where the wavelength of fluorescence is close to that of the ECL signal:

$$
\begin{gathered}
\mathrm{A}+\mathrm{e}^{-} \rightarrow \mathrm{A}^{-} \\
\mathrm{A}^{-}+\mathrm{BPO} \rightarrow \mathrm{A}+\mathrm{BPO}^{-} \\
\mathrm{BPO}^{-} \rightarrow \mathrm{C}_{6} \mathrm{H}_{5} \mathrm{CO}_{2}^{-}+\mathrm{C}_{6} \mathrm{H}_{5} \mathrm{CO}_{2} \\
\mathrm{~A}^{-}+\mathrm{C}_{6} \mathrm{H}_{5} \mathrm{CO}_{2} \rightarrow \mathrm{A}^{*}+\mathrm{C}_{6} \mathrm{H}_{5} \mathrm{CO}_{2}^{-}
\end{gathered}
$$

The thiophene dimer-2 produces only a small annihilation ECL signal because of the instability of the radical cation, which is produced in only small amounts. The resulting ECL emission is substantially red shifted compared to the PL; presumably, this is due to emission of a coupling product (Fig. 10a). The shift of the ECL emission is $100 \mathrm{~nm}$ less than that of the t-dimer-1 and the ECL maximum is at a shorter wavelength than the fluorescence of the t-tetramer. The ECL wavelength was the same in the presence of benzoyl peroxide suggesting absence of dimerization or further oligomerization with t-dimer-2. The trimers produce stronger ECL signals with a close agreement with the PL (Fig.$\mathrm{S} 16 \mathrm{a}, \mathrm{b} \dagger)$. There is a further increase in the ECL signal with chain length, e.g., for the t-tetramer. Presumably, this reflects the greater relative stability of the radical ions, as well as a general increase in the fluorescence quantum yield (Fig. 10b).

The ECL signal observed for $0.05 \mathrm{mM}$ t-hexamer-1 (Fig. 10c), demonstrates that it is possible to obtain ECL spectra even in cases where low solubility of the compounds precludes the generation of a substantial CV response. The t-hexamer-3, with long, branched alkyl substituents in the $\alpha$-positions, is characterized by the presence of shorter wavelength emission features, which disappear with addition of BPO (Fig. 10d,e). These spectral features probably reflect some formation of aggregates that are stabilized by the long alkyl chains, as seen previously in other systems. ${ }^{83}$ The radical cations are unstable in the absence of $\alpha$-substituents (e.g., t-hexamer-2, t-octamer, t-nonamer and t-dodecamer) and good ECL spectra cannot be obtained through annihilation (Fig. S16c-f $\dagger$ ). However with BPO as a coreactant, measurable signals were obtained. A strong ECL signal corresponding to the PL was observed for the t-dodecamer and ECL spectra could be obtained even with 20 pM solutions (Fig. 10f,g). The intensity of this signal, albeit obtained in a nonaqueous solvent, is close to that observed in aqueous media for $\mathrm{Ru}(\mathrm{bpy})_{3}{ }^{2+}$, used commercially for aqueous biological assays. ${ }^{84-88}$ The dodecamer produced much brighter ECL signal than the corresponding dimer, trimer or hexamer, in agreement with the trend in the fluorescence data noted above (Tables 1 and 2). These results indicate that any decrease in the ECL signal because of a decrease in diffusion coefficient in the range of molecular weights from dimer to dodecamer, is comparatively smaller than the effect of increased fluorescence efficiency. This increase appears to be related to the extent of delocalization of the electron density. In contrast to the oligothiophenes, the fluorene oligomers show ECL spectra characterized by a high efficiency even for the trimer (Table 2, Fig. 10hj). Here, however, an increase in chain length did not lead to an enhancement in the ECL intensity and, in fact, a small decrease in the ECL signal was seen (Table 2).

\section{Conclusions}

A series of electrochemical studies revealed the presence of multiple electron transfer steps for oligomers of thiophenes as well as those of fluorene. This conclusion was found to hold true over a wide range of samples and chain length sizes. The separation between sequential waves was attributed to the structures of the materials. The thiophene oligomers were characterized by a larger separation between the electrochemical waves for both oxidation and reduction as compared to the corresponding fluorenes of the same chain length, a difference that may be due to a higher extent of conjugation and smaller size relative to the bulky fluorene molecules as compared to the thiophenes. In all cases, it was found to be more difficult to add or remove a third electron as compared to a second electron, an effect ascribed to increasingly significant electrostatic effects. Thiophene and fluorene conjugated oligomers also showed strong ECL signals, attributed to the formation of stable radical cations and anions. The intensity of these signals increased with chain length for the thiophenes, but not the fluorenes. The strong ECL emission, especially with the larger oligothiophenes, suggested to us that these systems could find use in ECL-based applications. Formation of larger oligomers during ECL reactions has also been observed, demonstrating that ECL can be a useful technique for monitoring the ability of the molecules to form larger molecules from the observed emission spectrum.

\section{Acknowledgements}

We thank the Center of Electrochemistry, the program "Understanding Charge Separation and Transfer at Interfaces in Energy Materials (EFRC:CST)", an Energy Frontier Research Center funded by the Department of Energy (DOE) and the Robert A. Welch Foundation (F-0021, F-1018, and F-1621 for $\mathrm{AJB}, \mathrm{JLS}$, and CWB, respectively) for the support of this research. Salary support for DML for the synthesis of the $\mathbf{t}$ hexamer-2, t-octamer, t-nonamer and t-dodecamer was provided by the National Science Foundation CHE-01057904 (JLS).

\section{Notes and references}

1 T. Saji, N. E. Pasch, S. E. Webber and A. J. Bard, J. Phys. Chem., 1978, 82, 1101-1105.

2 T. W. Smith, J. E. Kuder and D. Wychick, J. Polym. Sci., 1976, 14, $2433-2448$.

3 J. B. Flanagan, S. Margel, A. J. Bard and F. C. Anson, J. Am. Chem. Soc., 1978, 100, 4248-4253.

4 A. F. Diaz, J. L. Castillo, J. A. Logan and W. Y. Lee, J. Electroanal. Chem., 1981, 129, 115-132.

5 M. Dietrich, J. Heinze, G. Heywang and F. Jonas, J. Electroanal. Chem., 1994, 369, 87-92.

6 C. P. Andrieux, P. Audebert, P. Hapiot and J. M. Saveant, J. Phys. Chem., 1991, 95, 10158-10164.

7 A. F. Diaz, K. K. Kanazawa and G. P. Gardini, J. Chem. Soc., Chem. Commun., 1979, 635-636.

8 A. Bettelheim, B. A. White, S. A. Raybuck and R. W. Murray, Inorg. Chem., 1987, 26, 1009-1017.

9 J. Heinze, J. Mortensen, K. Müllen and R. Schenk, J. Chem. Soc., Chem. Commun., 1987, 701-703.

10 J. Heinze, M. Störzbach and J. Mortensen, Ber. Bunsenges. Phys. Chem., 1987, 91, 960-967.

11 A. B. Nepomnyashchii, M. Bröring, J. Ahrens and A. J. Bard, J. Am. Chem. Soc., 2011, 133, 8633-8645.

12 D. Astruc, C. Ornelas and J. Ruiz, Chem.-Eur. J., 2009, 15, 89368944. 
13 P. Baüerle, U. Mitschke, G. Gruner and G. Rimmel, Pure Appl. Chem., 1999, 71, 2153-2160.

14 J. Guay, P. Kasai, A. Diaz, R. Wu, J. M. Tour and L. H. Dao, Chem. Mater., 1992, 4, 1097-1105.

15 P. Hapiot, C. Lagrost, F. Le Floch, E. Raoult and J. Rault-Berthelot, Chem. Mater., 2005, 17, 2003-2012.

16 S. Rihn, S. M. Erdem, A. De Nicola, P. Retailleau and R. Ziessel, Org. Lett., 2011, 13, 1916-1919.

17 K. Meerholz and J. Heinze, Electrochim. Acta, 1996, 41, 1839-1854.

18 J. Heinze, P. Tschuncky and A. Smie, J. Solid State Electrochem., 1998, 2, 102-109.

19 J. Heinze, B. A. Frontana-Uribe and S. Ludwigs, Chem. Rev., 2010, 110, 4724-4771.

20 H. Shirakawa, E. J. Louis, A. G. MacDiarmid, C. K. Chiang and A. J. Heeger, J. Chem. Soc., Chem. Commun., 1977, 578-580.

21 C. K. Chiang, Y. W. Park, A. J. Heeger, H. Shirakawa, E. J. Louis and A. J. MacDiarmid, Phys. Rev. Lett., 1977, 39, 1098-1101.

22 Q. Zhou and T. M. Swager, J. Am. Chem. Soc., 1995, 117, 7017-7018.

23 C. Li, M. Numata, M. Takeuchi and S. Shinkai, Angew. Chem., Int. Ed., 2005, 44, 6371-6374.

24 C. Fan, K. W. Plaxco and A. J. Heeger, J. Am.Chem. Soc., 2002, 124, $5642-5643$.

25 F. Hide, M. A. Diaz-Garcia, B. J. Schwartz and A. J. Heeger, Acc. Chem. Res., 1997, 30, 430-436.

26 M. Liu, R. Rieger, C. Li, H. Menges, M. Kastler, M. Baumgarten and K. Müllen, ChemSusChem, 2010, 3, 106-111.

27 R. H. Friend, R. W. Gymer, A. B. Holmes, J. H. Burroughes, R. N. Marks, C. Taliani, D. D. C. Bradley, D. A. Dos Santos, J.-L. Bredas, M. Logdlund and W. R. Salaneck, Nature, 1999, 397, 121-128.

28 P. F. Barbara, A. J. Gesquiere, S.-J. Park and Y. Lee, Acc. Chem. Res., 2005, 38, 602-610.

29 R. E. Palacios, F.-R. F. Fan, A. J. Bard and P. F. Barbara, J. Am. Chem. Soc., 2006, 128, 9028-9029.

30 R. E. Palacios, F.-R. F. Fan, J. K. Grey, J. Suk, A. J. Bard and P. F. Barbara, Nat. Mater., 2007, 6, 680-685.

31 D. Hu, J. Yu, K. Wong, B. Bagchi, P. J. Rossky and P. F. Barbara, Nature, 2000, 405, 1030-1033.

32 Y. Geng, A. Trajkovska, D. Katsis, J. J. Ou, S. W. Culligan and S. H. Chen, J. Am. Chem. Soc., 2002, 124, 8337-8347.

33 R. Anemian, J.-C. Mulatier, C. Andraud, O. Stephan and J.-C. Vial, Chem. Commun., 2002, 1608-1609.

34 H. Nakanishi, Y. Aso and T. Otsubo, Synth. Met., 1999, 101, 604 605 .

35 Y. Ie, A. Han, T. Otsubo and Y. Aso, Chem. Commun., 2009, 30203022.

36 A. Kreyes, M. Amirkhani, I. Liebirwith, R. Mauer, F. Laquai, K. Landfester and U. Ziener, Chem. Mater., 2010, 22, 6453-6458.

37 R. S. Becker, J. S. De Melo, A. L. Macanita and F. Elisei, J. Phys. Chem., 1996, 100, 18683-18695.

38 J. P. Choi, K.-T. Wong, Y.-M. Chen, J.-K. Yu, P.-T. Chou and A. J. Bard, J. Phys. Chem. B, 2003, 107, 14407-14413.

39 C. Poriel, J. Rault-Berhelot, D. Thirion, F. Barriere and L. Vignau, Chem.-Eur. J., 2011, 17, 14031-14046.

40 M. G. Hill, K. R. Mann, L. L. Miller and J.-F. Penneau, J. Am. Chem. Soc., 1992, 114, 2728-2730.

41 M. G. Hill, J.-F. Penneau, B. Zinger, K. P. Mann and L. L. Miller, Chem. Mater., 1992, 4, 1106-1113.

42 J. Suk, K. M. Omer, T. Bura, R. Ziessel and A. J. Bard, J. Phys. Chem. C, 2011, 115, 15361-15368.

43 E. Fabrizio, I. Prieto and A. J. Bard, J. Am. Chem. Soc., 2000, 122, 4996-4997.

44 A. B. Nepomnyashchii, M. Bröring, J. Ahrens and A. J. Bard, J. Am. Chem. Soc., 2011, 133, 19498-19504.

45 S. K. Lee, M. M. Richter, L. Strekowski and A. J. Bard, Anal. Chem., 1997, 69, 4126-4133.

46 S. Stas, S. Sergeyev and Y. Geertz, Tetrahedron, 2010, 66, 1837-1845.

47 P. Bäuerle, F. Würthner, G. Götz and F. Effenberger, Synthesis, 1993, 11, 1099-1103.

48 G. Barbarella, L. Faveretto, G. Sotgiu, M. Zambianchi, L. Antolini, O. Pudova and A. Bongini, J. Org. Chem., 1998, 63, 5497-5506.

49 J.-M. Raimundo, E. Levillain, N. Galego-Planas and J. Roncali, Electrochem. Commun., 2000, 2, 211-215.
50 H. Kanato, K. Takimiya, T. Otsubo, Y. Aso, T. Nakamura, Y. Araki and O. Ito, J. Org. Chem., 2004, 69, 7183-7189.

51 J. A. Riddick and W. B. Bunger, Organic Solvents: Physical Properties and Methods of Purification, Wiley, New York, 1986.

52 M. Lu, S. Nagamatsu, Y. Yoshida, M. Chikamitsu, R. Azumi and K. Yase, Chem. Lett., 2010, 39, 60-61.

53 D. E. Jansen, M. W. Burand, P. C. Ewbank, T. M. Pappenfus, H. Higuchi, A. da Silva Filho, V. G. Young, J.-L. Bredas and K. P. Mann, J. Am. Chem. Soc., 2004, 126, 15295-15308.

54 M. Sato and M. Hiroi, Chem. Lett., 1994, 6, 985-988.

55 J. H. Brannon and D. Magde, J. Phys. Chem., 1978, 82, 705-709.

56 S. Sahami and M. Weaver, J. Electroanal. Chem., 1981, 122, 155-170.

57 M. Rudolph, J. Electroanal. Chem., 1991, 314, 13-22.

58 M. Rudolph, J. Electroanal. Chem., 1992, 338, 85-98.

59 J. Mocak and S. W. Feldberg, J. Electroanal. Chem., 1997, 378, 31-37.

$60 \mathrm{~S}$. W. Feldberg, C. I. Goldstein and M. Rudolph, J. Electroanal. Chem., 1996, 413, 25-36.

61 M. Belletete, L. Mazorolle, N. Desrosiers, M. Leclerc and G. Durocher, Macromolecules, 1995, 28, 8587-8597.

62 A. Yassar, D. Delabouglise, M. Hmyene, B. Nessak, G. Horowitz and F. Garnier, Adv. Mater., 1992, 4, 490-494.

63 P. F. Van Hutten, R. E. Gill, J. K. Herema and G. Hadziioannou, J. Phys. Chem., 1995, 99, 3218-3224.

64 L. DeWitt, G. J. Blanchard, E. LeGoff, M. E. Benz, J. H. Liao and M. G. Kanatzidis, J. Am. Chem. Soc., 1993, 115, 12158-12164.

65 J. C. Horne, G. J. Blanchard and E. LeGoff, J. Am. Chem. Soc., 1995, 117, 9551-9558.

66 K.-T. Wong, Y.-Y. Chien, R.-T. Chen, C.-F. Wang, Y.-T. Lin, H.-H. Chiang, P. J. Hsieh, C.-C. Wu, C. H. Chou, Y. O. Su, G.-H. Lee and S.-M. Peng, J. Am. Chem. Soc., 2002, 124, 1157611577.

67 U. Mitschke and P. Bäuerle, J. Chem. Soc., Perkin Trans. 1, 2001, 1, 740-753.

68 A. J. Bard and L. Faulkner, Electrochemical Methods: Fundamentals and Applications; Second Edition; John Wiley and Sons: New York, 2001.

69 P. Hapiot, P. Audebert, K. Monnier, J. M. Pernaut and P. Garcia, Chem. Mater., 1994, 6, 1549-1555.

70 A. Fachetti, M.-H. Yoon, C. L. Stern, G. R. Hutchison, M. A. Ratner and T. J. Marks, J. Am. Chem. Soc., 2004, 126, 13480-13501.

71 S. S. Zade and M. Bendnikov, J. Phys. Chem. B, 2006, 110, 1583915846.

72 S. S. Zade and M. Bendnikov, J. Phys. Chem. C, 2007, 111, $10662-$ 10672.

73 D. Beljonne, J. Cornil, R. H. Friend, R. A. Janssen and J.-L. Bredas, J. Am. Chem. Soc., 1996, 118, 6453-6461.

74 J.-L. Bredas, R. Silbey, D. S. Boudreaux and R. R. Chance, J. Am. Chem. Soc., 1983, 105, 6555-6559.

75 T. Nishinaga, D. Yamazaki, M. Tateno, M. Iyoda and K. Komatsu, Materials, 2010, 3, 2037-2052.

76 C. Lin, T. Endo, M. Takase, M. Iyoda and T. J. Nishinaga, J. Am. Chem. Soc., 2011, 133, 11339-11350.

77 F. Fungo, K.-T. Wong, S. Y. Ku, Y.-Y. Hug and A. J. Bard, J. Phys. Chem. B, 2005, 109, 3984-3989.

78 M. Shen, J. Rodriguez-Lopez, Y.-T. Lee, C.-T. Chen, F.-R. Fan and A. J. Bard, J. Phys. Chem. C, 2010, 114, 9772-9780.

79 K. M. Omer, S. Y. Ku, K.-T. Wong and A. J. Bard, J. Am. Chem. Soc., 2009, 131, 10733-10741.

80 K. M. Omer, A. L. Kanibolotsky, P. J. Skabara, I. F. Perepichka and A. J. Bard, J. Phys. Chem. B, 2007, 111, 6612-6619.

81 L. Nadjo and J. M. Saveant, J. Electroanal. Chem., 1973, 44, 327366.

82 J. M. Saveant, Elements of Molecular and Biomolecular Electrochemistry; Wiley-Interscience: Hoboken, 2006, 102-106.

83 A. B. Nepomnyashchii, M. Bröring, J. Ahrens, R. Krüger and A. J. Bard, J. Phys. Chem. C, 2010, 114, 14453-14460.

84 N. E. Tokel and A. J. Bard, J. Am. Chem. Soc., 1972, 94, 2862-2863.

85 I. Rubinstein and A. J. Bard, J. Am. Chem. Soc., 1981, 103, 512-516.

86 N. E. Tokel-Takvoryan, R. E. Hemingway and A. J. Bard, J. Am. Chem. Soc., 1973, 95, 6582-6589.

87 W. Miao, J.-P. Choi and A. J. Bard, J. Am. Chem. Soc., 2002, 124, $14478-14485$.

88 H. White and A. J. Bard, J. Am. Chem. Soc., 1982, 104, 6891-6895. 